\title{
Measuring the yield stress in magnetorheological fluids using ultrasounds
}

Jaime Rodríguez-López ${ }^{1}$, Luis Elvira ${ }^{1}$, Francisco Montero de Espinosa Freijo ${ }^{1}$, Georges Bossis ${ }^{2}$, Juan de Vicente ${ }^{3}$

${ }^{1}$ Spanish National Research Council. Centre of Applied Physics and Non Destructive Evaluation. (CAEND-CSIC), 28006-Madrid, Spain

${ }^{2}$ Laboratoire de Physique de la Matière Condensée, Université de Nice Sophia Antipolis, CNRS UMR 7663, Parc Valrose, 06108 Nice Cedex 2, France

${ }^{3}$ Department of Applied Physics, Faculty of Sciences, University of Granada, 18071Granada, Spain

Email: jvicente@ugr.es

\begin{abstract}
In this work, we propose a new method to accurately determine the yield stress in magnetorheological (MR) fluids using ultrasounds. The setup is constructed and experimental data are obtained on a model conventional MR fluid under steady shear stress ramp-up tests. By using video-microscopy, ultrasonic techniques and rheometry, simultaneously, it is possible to precisely determine the yield stress at experimentally accessible times.
\end{abstract}

\section{Keywords}

Magnetorheology, magnetorheological fluids, yield stress, ultrasounds. 
Measuring the yield stress of pasty materials is a long-term challenge in rheology. This is so probably because even today there is not a consensus on the existence of a true yield stress (Barnes, 1999; Moller et al., 2006; Berli and de Vicente, 2012). Bearing this in mind, it is not difficult to understand that there is not a unique and precise method to determine the yield point. In this letter we demonstrate that the use of ultrasounds is of valuable help in the determination of the yield point, especially in the case of magnetic field-responsive colloids --magnetorheological (MR) fluids-- where the yield stress can be tuned externally. However, this approach can also be extended to the study of the yielding behavior of other pasty materials such as colloidal gels and glasses. To our knowledge, no previous attempts have been done in the past to combine shear rheometry and longitudinal ultrasonic characterization for the determination of the yield stress.

There are several approaches to determine the yield stress of MR fluids. Essentially, all of them can be classified in two groups: direct and indirect methods. Direct methods involve the use of creep tests (Li et al., 2002; Berli and de Vicente, 2012), stress ramp tests (Bombard et al., 2002; Ulicny and Golden, 2007; Yang et al., 2009; Jonkkari and Syrjala, 2010), and stress growth tests (Ulicny and Golden, 2007; Jonkkari and Syrjala, 2010). Indirect approaches are most frequently used in the literature and are based on the low-shear extrapolation of flow curves (stress versus shear rate) both in lin-lin or log-log representations (de Vicente et al., 2011; Ulicny et al., 2010). More recently, dynamic oscillatory amplitude sweep experiments are being used to determine the yield point either from the appearance of high order harmonics in the mechanical signal and the concomitant rapid decrease in the storage modulus versus stress curves (i.e. onset of non-linearity) (de Vicente et al., 2002; Laun et al., 2009), the crossover between the storage and loss moduli (Segovia-Gutierrez et al., 2012), or the maximum in the elastic stress (Pan and McKinley, 1997; Segovia-Gutierrez et al., 2012). The yield values determined from direct and indirect approaches are sometimes referred to as the static and dynamic yield stress definitions, respectively.

In previous works, magnetorheometry, in dilute systems, has been complemented with the use of optical microscopy as the dispersed particles, and field-induced aggregates, have typical sizes of the orders of microns or larger (Volkova et al., 1999; Claracq et al., 2004). However, MR fluids that are of interest in commercial applications do contain 
exceedingly large amounts of dispersed iron particles (approx. $30 \mathrm{vol} \%$ ). At these concentrations MR fluids are opaque to the light and as a result, the use of rheo-optical and scattering techniques is precluded. Also important is the fact that the yielding behavior of these concentrated systems becomes far to be trivial. In some cases thixotropy, particle migration, shear-banding and two-step yielding phenomena have been documented in the literature. In this sense, other non-intrusive techniques are needed.

A priori, low intensity ultrasonic characterization seems to be an interesting candidate technique to interrogate the yielding behavior of MR fluids providing an in situ characterization of the materials' microstructure under deformation. In fact, this technique is sensitive to changes in the viscoelastic properties of materials and it can be used to inspect opaque materials (Elvira et al., 2009; Lynnworth, 1975; Sierra et al., 2004).

With this aim, a novel experimental setup was built (see Figure 1) that combines the use of video-microscopy and ultrasounds together with rheometry. We employed a rotational stress-controlled Anton Paar MCR 501 rheometer in parallel plate configuration (diameter $65 \mathrm{~mm}$ ). The upper (rotating) plate $(\mathrm{Pu})$ was made of glass while the lower (stationary) plate $(\mathrm{Pb})$ was made of PMMA. Experiments were run in isothermal conditions $\left(25^{\circ} \mathrm{C}\right)$ using a Julabo circulator bath (TB) and a P-PTD 200 Peltier system (p). The magnetic field was generated through a custom built coil that generates a uniform magnetic field strength of approximately $15 \mathrm{kA} / \mathrm{m}$ within the region occupied by the sample. The field presents a maximum deviation from the center of $\pm 5 \%$ in the radial direction. This magnetic field strength was large enough to induce a sol-gel transition in the MR fluids (Segovia-Gutierrez et al., 2012). A LU165C-IO Lumenera CCD camera was adapted to a Mitutoyo objective (M) with $20 \times$ magnification and placed below the bottom plate to take pictures of the stressed sample. The acquisition of the snapshots was synchronized with the rheological and acoustic data. The ultrasonic block consisted of an ultrasonic transducer (T) placed into a cylindrical hole made on the rheometer bottom plate leaving the transducer emitting face, or the delay line if it is used, in contact with the sample. The transducer worked in pulse echo using the upper rheometer plate as reflector. An ultrasonic generator Parametrics UA 520 was used (P) to excite the transducer. The received signals were acquired by a Tektronix TDK210 (O) 
oscilloscope and recorded in a computer (PC) via GPIB. Time of flight variations (TOF, i.e. the time which takes the longitudinal ultrasonic wave to travel the thickness sample) and amplitude variations of the acoustic wave are calculated from the acquired signals (Rodriguez-Lopez et al., 2012). Both the transducer and the microscope objective were placed at the same radial position $(11.5 \mathrm{~mm})$.

Some constraints on the distance between the shearing surfaces had to be taken into account. The distance between the shearing surfaces, that defines the sample thickness, had to be small enough to avoid the ejection of the sample when being subjected to high shear stresses and to prevent non-homogeneity in the magnetic field. Also, it had to be large enough to decrease the uncertainty related with the inherent misalignment of the plates. Regarding the sound attenuation, the gap distance had to remain small as the amplitude of the reflected waves decays exponentially with distance, but also, it had to be high enough to avoid the interference of reflected echoes. A distance of $300 \mu \mathrm{m}$ was used in the conducted experiments as a compromise to agree with these constraints.

As mentioned above, the resonant frequency and bandwidth of the transducer had to be chosen carefully according to the distance between the plates and the sample acoustic attenuation. The pulses had to be short enough to get clean and isolated reflected signals; therefore, high frequency broad band transducers were used. However, the sound attenuation in the sample increased with frequency and so if the emitted frequency was very high, the acoustical signal would be too weak to obtain good signal to noise ratio. A compromise had to be found. Accordingly, a Doppler DL15P6T -Doppler, Chinalongitudinal waves transducer with a central frequency of $15 \mathrm{MHz}$ with $70 \%$ of bandwidth was chosen.

Model MR fluids were formulated by dispersing (large) carbonyl iron microparticles (OM grade from BASF SE, mean diameter 5 microns) in a highly viscous silicone oil (487 mPa.s, Sigma-Aldrich) so as to prevent significant sedimentation during the experiments. To keep the fluids as simple as possible, we decided not to include further additives in their formulation. The particle content was fixed at 5 vol\%. This concentration was low enough in order to make it possible the visualization of the structures under shear. At the same time, the concentration had to be large enough to get a non-Newtonian behavior due to the presence of the field-induced structures. 
Preliminary experiments (not shown here for brevity) demonstrated that these MR fluids behaved as non-thixotropic model yield stress materials.

The whole experiments consist in six different stages, although in this work, we are mainly interested in the forth one. As a way of example, Figure 2, shows TOF, amplitude and shear rate data acquired as a function of time during the complete experiment under the presence of an external magnetic field. Experiments started with a preconditioning protocol at $100 \mathrm{~s}^{-1}$ during $100 \mathrm{~s}$ to erase any memory effects of the suspensions (I). Thereupon, the sample was left to equilibrate at rest during another 100 s (II). Next, a DC magnetic field (approx. $15 \mathrm{kA} / \mathrm{m}$ ) was suddenly applied, if needed, during $100 \mathrm{~s}$ to promote the field-induced structuration (III). Then, the shear stress was logarithmically increased from $0.1 \mathrm{~Pa}$ to $1000 \mathrm{~Pa}$ at a rate of $5 \mathrm{~s}$ per data point still in the presence of the magnetic field (IV). After that, the stress was removed and the sample remained at rest during $50 \mathrm{~s}$ in the presence of the magnetic field (V). Finally, the magnetic field was switched off and the data recorded for another $50 \mathrm{~s}$ (VI). During the whole test, both the CCD camera and the ultrasound probe were switched on to record data.

Results obtained during the shear stress ramp interval (interval IV in Figure 2) are shown in Figures 3 and 4 both in the absence and presence of magnetic fields. Figure 3 reveals that, even in the off-state, the suspension exhibits a non-Newtonian (shearthinning) behavior for the time scales under investigation. For low enough apparent rim shear stresses the suspension appears to be arrested and the particles are aggregated due to the existence of short-ranged interparticle forces coming from van der Waals forces and/or some remnant magnetization within the particles. If truly exists, the yield stress value is expected to depend on the time scale under investigation, and in particular in the time employed to measure every point in Figure 3.

Measuring at low shear rates requires long acquisition times. As a practical rule, the time required for achieving the steady state at a given shear rate should be at least larger than the reciprocal of the shear rate. As a consequence, rheograms reported in the literature are in most cases only worth to be trusted for shear rates larger than approximately $0.1 \mathrm{~s}^{-1}$. Generally speaking, data corresponding to lower shear rates are not necessarily at steady state, and therefore the use of acoustic probes is of valuable 
help. From the inspection of Figure 3 we clearly see that in contrast to rheological material functions that smooth and gradually change with increasing the stress, the signal amplitude and TOF, typically experience drastic changes close to where we envisage the yield point.

In the absence of magnetic fields (see Figure 3), both the signal amplitude and TOF are constant at low stress levels, due to the fact that the microstructure does not change and remains unperturbed $(3 \mathrm{~A})$. However, upon increasing the apparent rim shear stress, the TOF starts to increase, presumably due to the breakage of interaggregate bonds well before the complete yielding of the structures $(3 \mathrm{~A}-3 \mathrm{E})$. These results are in agreement with the Harker and Temple suspension model (Harker and Temple, 1988). If the stress increases a little bit more, the amplitude experiences a drastic reduction while the TOF reaches a local maximum, being at this stage where the MR fluid is expected to start flowing. Optical microscope observations do corroborate these hypotheses (3E-3I). In summary, as demonstrated in the snapshots contained in Figure 3, the structure remains unaltered up to stresses close to $5 \mathrm{~Pa}(3 \mathrm{~A})$. Then, some destructuring occurs while the TOF increases (3A-3E), and finally at $8 \mathrm{~Pa}(3 \mathrm{E})$, the suspension fully flows when the amplitude drops and the TOF reaches a maximum.

In the presence of magnetic fields, particles interact and rearrange forming elongated structures. Therefore, in this case, the scenario is markedly different than before (see Figure 4). Similar to the case where the magnetic field is not applied, both acoustic magnitudes remain initially constant at low stress levels. This is coherent with microscopic images that do not evolve with time (4A). Then, above approximately 10 $\mathrm{Pa}$, both the amplitude and TOF change; the former increases with stress, while the later decreases. In this region, structures are evolving as demonstrated by the photographs $(4 \mathrm{~A}-4 \mathrm{H})$. The structures are reforming during time remaining as elongated fibers but with a larger diameter as a result of the fragmentation effect of the shearing forces and the reforming effect of the magnetic field. Accordingly to Ahuja's model (Ahuja and Hendee, 1978), if fiber-like structures have a larger diameter, signal amplitude must increase and TOF must decrease, being in agreement with the experimental results. Furthermore, this structure thickening effect does provide another source of increasing the stiffness due to the rearrangement of particles in agreement with the acoustical 
behavior shown. At $80 \mathrm{~Pa}(4 \mathrm{H})$, the amplitude reaches a maximum and the TOF reaches a minimum. These findings are associated to the onset of flow in the suspension (4H-4I).

In summary, in the absence of magnetic fields the signal amplitude remains almost constant up to the stress value where the sample starts to flow. On the contrary, in the presence of magnetic fields the signal amplitude starts to increase prior to the onset of flow due to fact that aggregates reform under shear. On the other hand, TOF increases prior to the onset of flow in the absence of magnetic fields. However, in the presence of fields, TOF decreases prior to the yield point in good agreement with the fact that structures reform before breaking at the yield point. This is coherent with a thicker columnar structure and hence a larger propagation speed that is confirmed with microscopic images.

We can distinguish a total of three regions in the apparent rim shear stress vs. shear rate curves for magnetized MR fluids. The first region occurs at exceedingly low stress values and is associated to a quasistatic structure (either a solid or a fluid with a very high viscosity). The second region is related to evolving structures where both hydrodynamics and magnetostatics play a role. At this stage both the acoustic signal amplitude and the shear rate increase while the TOF decreases. This is expected to be associated to the strengthening of the field-induced structures under shear in the search of low-energy states. Finally, a third region concerns very large stresses. In this hydrodynamic dominated region, acoustic signals become apparently chaotic and structures fully flow under shear.

To conclude, a new method is proposed to accurately determine the yield stress in pasty materials by measuring the longitudinal ultrasonic propagation under shearing forces. This approach is tested in the case of model MR fluids (with tunable yield stress) by simultaneously measuring optical, mechanical and acoustical properties of the suspensions.

This work was supported by MICINN MAT 2010-15101, I+D DPI2010-17716 and PIB2010BZ-00570 projects (Spain), and by Junta de Andalucía P10-RNM-6630 and P11-FQM-7074 projects (Spain). J.R.-L. acknowledges financial support by a CSIC JAE fellowship. 


\section{Reference list}

Ahuja A S and Hendee W R 1978 Effects of particle shape and orientation on propagation of sound in suspensions The Journal of the Acoustical Society of America 63 1074-80

Barnes H A 1999 The yield stress-a review Journal of Non-Newtonian Fluid Mechanics 81 133-78

Berli C L A and de Vicente J 2012 A structural viscosity model for magnetorheology Applied Physics Letters 101

Berli C L A and de Vicente J 2012 submitted

Bombard A J F, Knobel M, Alcantara M R and Joekes I 2002 Evaluation of Magnetorheological Suspensions Based on Carbonyl Iron Powders Journal of Intelligent Material Systems and Structures 13 471-8

Claracq J, Sarrazin J and Montfort J P 2004 Viscoelastic properties of magnetorheological fluids Rheologica Acta 43 38-49

de Vicente J, Gonzalez-Caballero F, Bossis G and Volkova O 2002 Normal force study in concentrated carbonyl iron magnetorheological suspensions Journal of Rheology 46 1295-303

de Vicente J, Klingenberg D J and Hidalgo-Alvarez R 2011 Magnetorheological fluids: a review Soft Matter 7 3701-10

Elvira L, Rodriguez J and Lynnworth L C 2009 Sound speed and density characterization of milk adulterated with melamine The Journal of the Acoustical Society of America 125 EL177-EL82

Harker A H and Temple J A G 1988 Velocity and attenuation of ultrasound in suspensions of particles in fluids Journal of Physics D: Applied Physics 21 1576-88

Jonkkari I and Syrjala S 2010 Evaluation of techniques for measuring the yield stress of a magnetorheological fluid Applied Rheology 2045875 
Laun H M, Gabriel C and Kieburg C H 2009 Magnetorheological fluid (MRF) in oscillatory shear and parameterization with regard to MR device properties Journal of Physics: Conference Series 149012067

Li W H, Du H, Chen G and Yeo S H 2002 Experimental investigation of creep and recovery behaviors of magnetorheological fluids Materials Science and Engineering: A 333 368-76

Lynnworth L C 1975 Industrial applications of ultrasound. A review. II. Measurements, tests, and process control using low intensity ultrasound IEEE Transactions on Sonics and Ultrasonics 54-22 71-101

Moller P C F, Mewis J and Bonn D 2006 Yield stress and thixotropy: On the difficulty of measuring yield stresses in practice Soft Matter 2 274-83

Pan X-D and McKinley G H 1997 Structural limitation to the material strength of electrorheological fluids Applied Physics Letters 71 333-5

Rodriguez-Lopez J, Elvira Segura L and Montero De Espinosa Freijo F 2012 Ultrasonic velocity and amplitude characterization of magnetorheological fluids under magnetic fields Journal of Magnetism and Magnetic Materials 324 222-30

Segovia-Gutierrez J P, Berli C L A and De Vicente J 2012 Nonlinear viscoelasticity and two-step yielding in magnetorheology: A colloidal gel approach to understand the effect of particle concentration Journal of Rheology 56 1429-48

Sierra C, Elvira L, Garcia J L, Resa P and Galan B 2004 Monitoring Escherichia coli growth in M63 media by ultrasonic noninvasive methods and correlation with spectrophotometric and HPLC techniques Applied Microbiology and Biotechnology 85 813-21

Ulicny J C and Golden M A 2007 Evaluation of yield stress meausrement techniques on a parallel plate magnetic rheometer International Journal of Modern Physics B 21 4898-906

Ulicny J C, Snavely K S, Golden M A and Klingenberg D J 2010 Enhancing magnetorheology with nonmagnetizable particles Applied Physics Letters 96 231903-6 
Volkova O, Cutillas S and Bossis G 1999 Shear Banded Flows and Nematic-toIsotropic Transition in ER and MR Fluids Physical Review Letters 82 233-6

Yang Y, Li L and Chen G 2009 Static yield stress of ferrofluid-based magnetorheological fluids Rheologica Acta 48 457-66 


\section{Figure legends}

FIG. 1. (Color online) Experimental set up where $C$ is the magnetic coil, $M$ the CCD camera adapted to the objective, $O$ is the oscilloscope, $P$ is the ultrasonic generator, $p$ is the Peltier system controlled by a thermal bath $(T B), P b$ is the bottom plate of the rheometer while $P u$ is the upper plate. $P C$ is the computer, $S$ the sample and $T$ the piezoelectric transducer.

FIG. 2. (Color online) Time of flight, amplitude and shear rate data as function of time during a complete experiment. A whole experiment consists of six different stages, although it is the fourth stage the one which provides the experimental results shown in this work. First, there is a preconditioning protocol at $100 \mathrm{~s}^{-1}$ during $100 \mathrm{~s}$ (I). Second, the sample was left to equilibrate at rest during another $100 \mathrm{~s}$ (II). Next, a DC magnetic field (approx. $15 \mathrm{kA} / \mathrm{m}$ ) was suddenly applied, if needed, during $100 \mathrm{~s}$ (III). Then, the shear stress was logarithmically increased from $0.1 \mathrm{~Pa}$ to $1000 \mathrm{~Pa}$ at a rate of $5 \mathrm{~s}$ per data point still in the presence of the magnetic field (IV). After that, the stress was removed in the presence of the magnetic field -if it was applied-for about $50 \mathrm{~s}(\mathrm{~V})$. Finally, the magnetic field was switched off and the data recorded for another $50 \mathrm{~s}$ (VI).

FIG. 3. (Color online) Time of flight, amplitude and shear rate as function of the apparent rim shear stress when no magnetic field is applied to the MR fluid (5 vol\%). Pictures from 3A to 3I show the microstructure at different stress levels.

FIG. 4. (Color online) Time of flight, amplitude and shear rate as function of the apparent rim shear stress when a magnetic field of $15 \mathrm{kA} / \mathrm{m}$ is applied to the MR fluid (5 vol\%). Pictures from 4A to 4I show the microstructure at different stress levels. 
Figures

FIG. 1

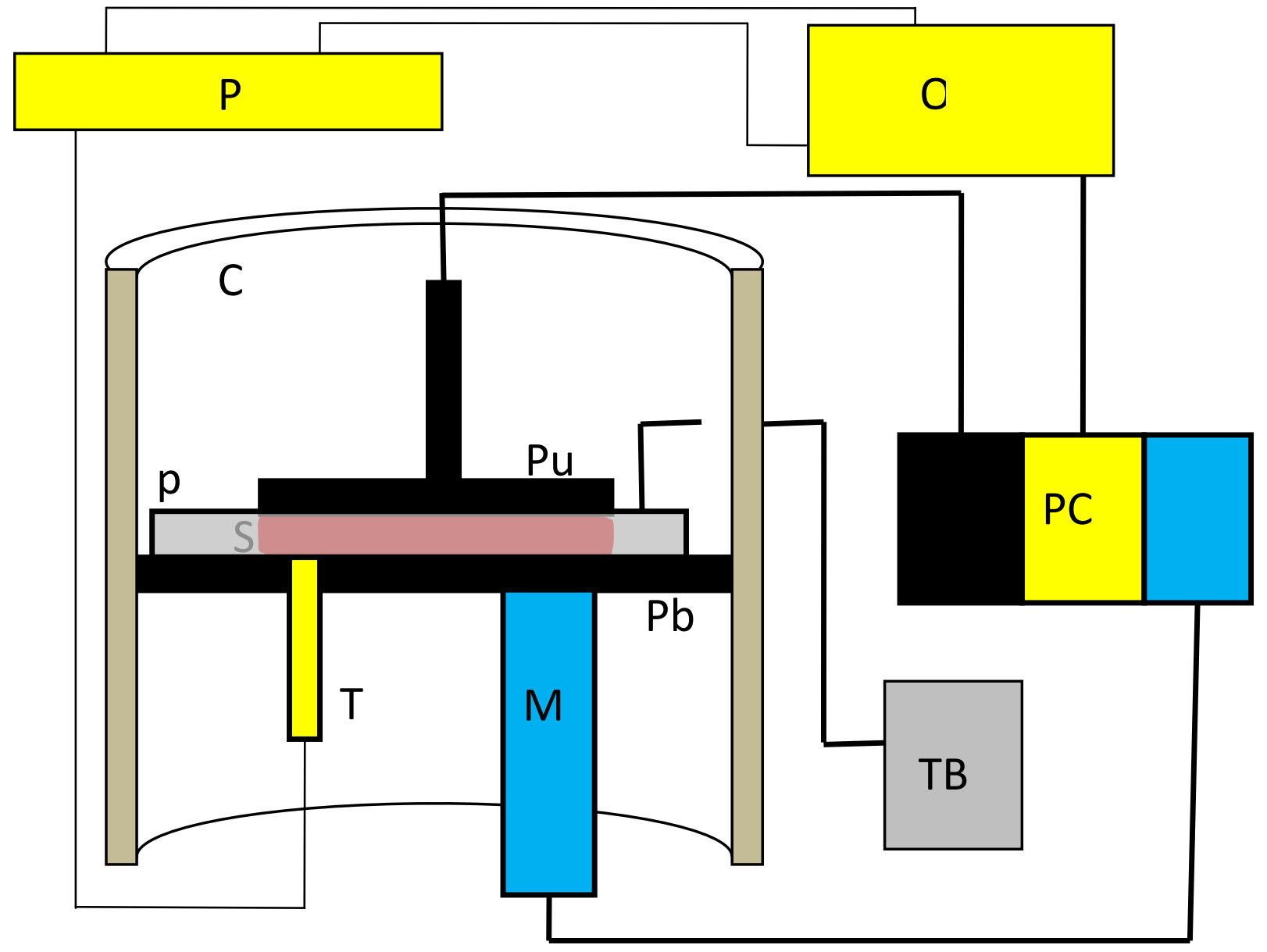


FIG. 2

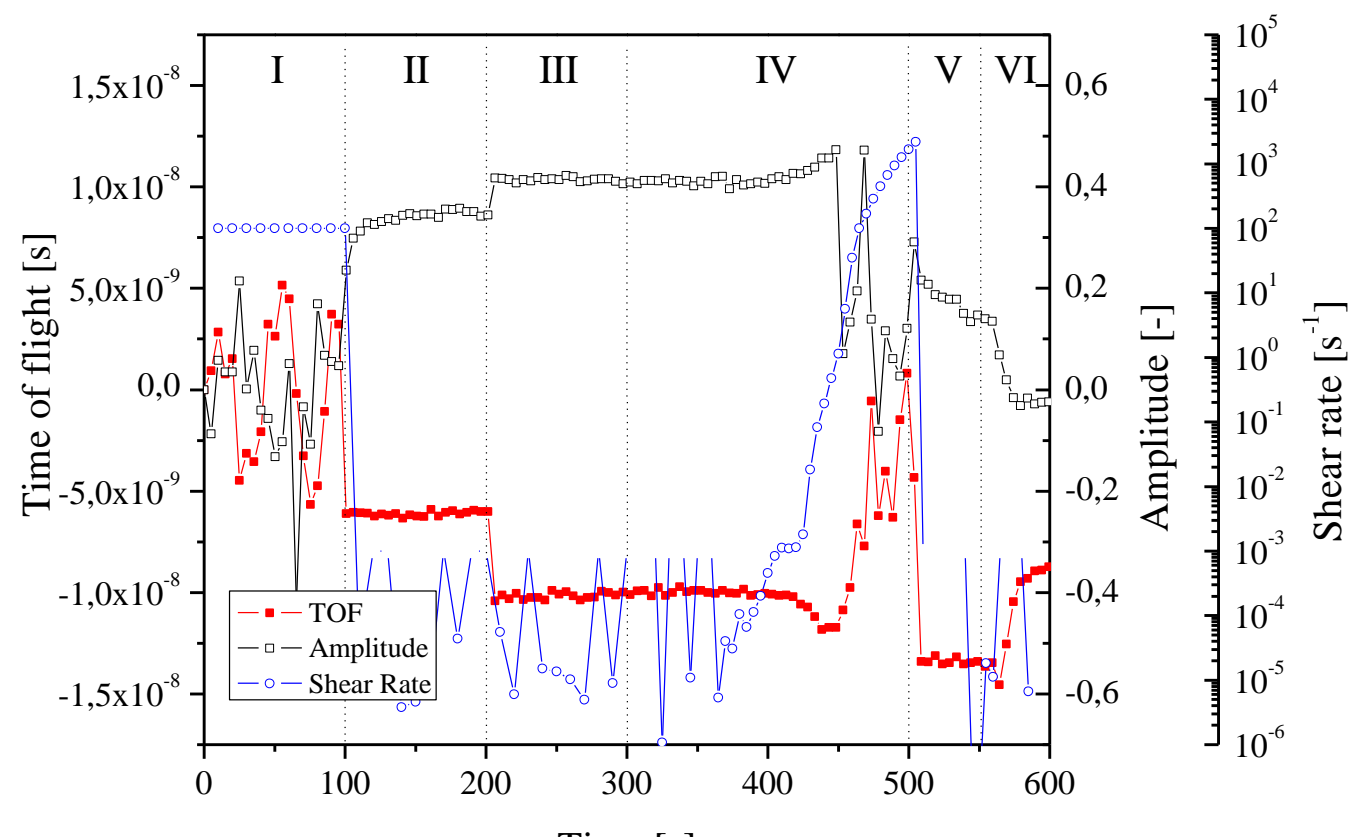

Time [s] 
FIG. 3
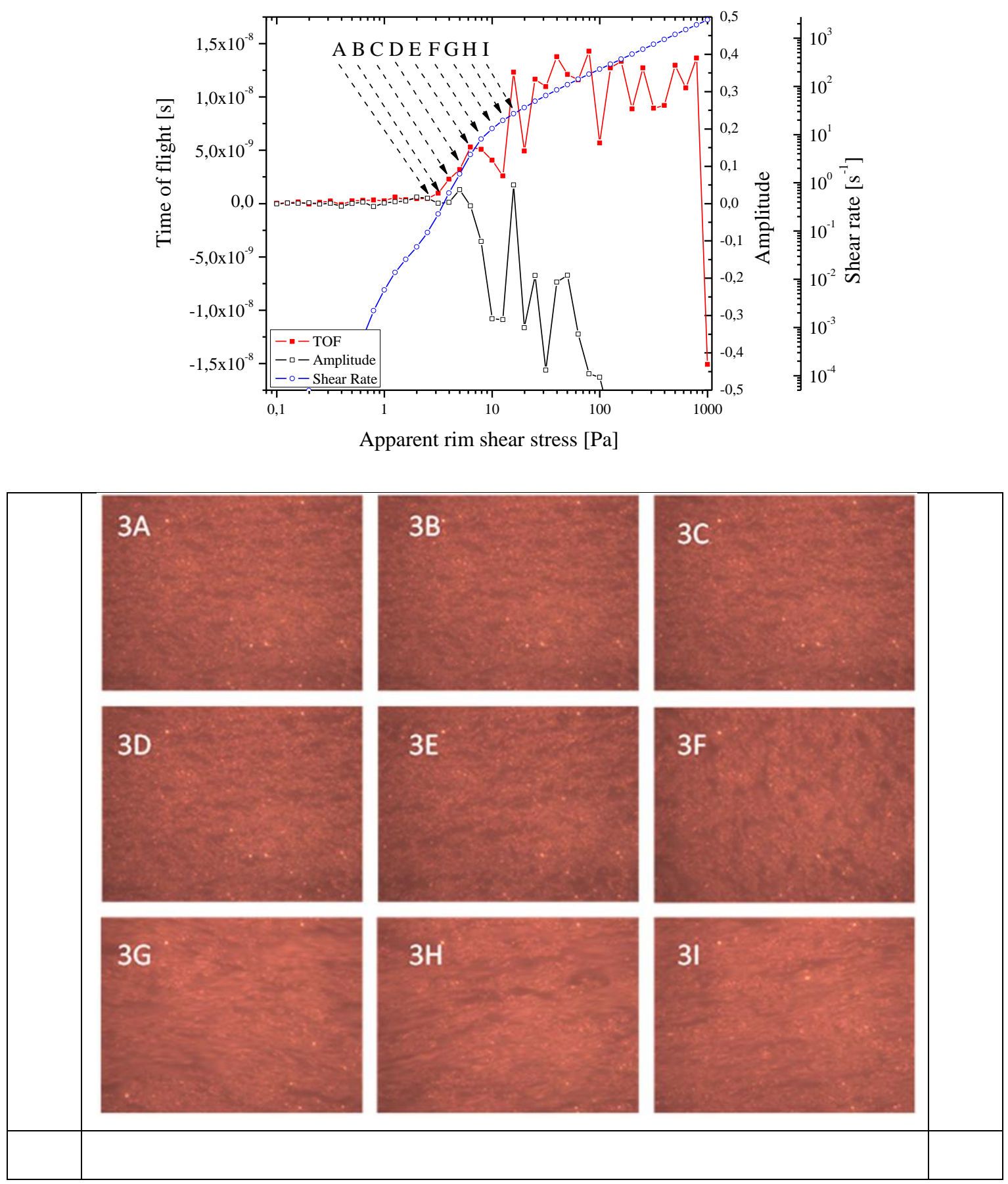
FIG. 4
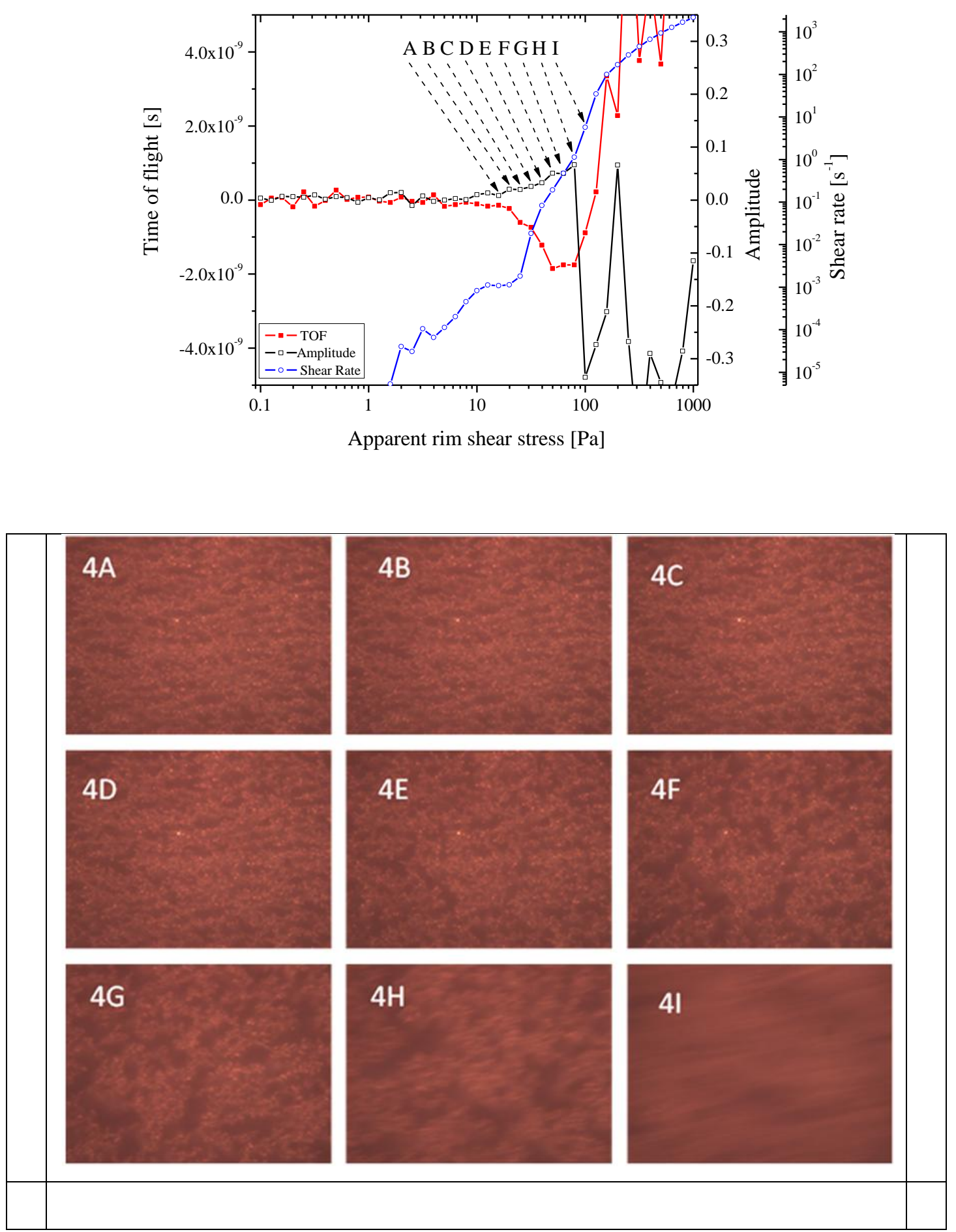